International Mathematical Forum, Vol. 9, 2014, no. 27, 1309 - 1319

HIKARI Ltd, www.m-hikari.com

http://dx.doi.org/10.12988/imf.2014.4598

\title{
Tensor Product of LA-Modules
}

\author{
Ahmad M. Alghamdi \\ Department of Mathematical Sciences \\ Faculty of applied Sciences \\ Umm Alqura University \\ P.O. Box 14035, Makkah 21955, Saudi Arabia \\ Fatiha Sahraoui \\ Department of Mathematics, Faculty of Sciences \\ Alqassim University, Buridah, Saudi Arabia \\ and \\ University Djillali Liabès of Sidi Bel Abbès \\ Department of mathematics, P. O. Box 89, 22000 \\ Sidi Bel Abbès, Algeria
}

Copyright (c) 2014 Ahmad M. Alghamdi and Fatiha Sahraoui. This is an open access article distributed under the Creative Commons Attribution License, which permits unrestricted use, distribution, and reproduction in any medium, provided the original work is properly cited.

\begin{abstract}
An LA-module over an LA-ring is defined in [19]. We define and construct a tensor product of two LA-modules. Although, LA-groups and LA-modules need not to be abelian, the new construction behaves like standard definition of the tensor product of usual modules over a ring. We then extend some simple results from the ordinary tensor to the new setting.
\end{abstract}

Mathematics Subject Classification: 20M25; 20N02; 16L30

Keywords: LA-modules; Tensor Product; Non-abelian tensor product; Semi-groups 


\section{Introduction}

The main motivation of the work in this paper is to generalize the concept of tensor product from the commutative case to the non-commutative case. It is well known that mathematicians define the tensor product for the vector spaces as well as of modules. For algebras over a field, mathematicians have done a lot of work in this direction (see page 229 in [5, Theorem 7.4, Chapter 4]). On the other hand, the definition of tensor product of non abalian groups is another story and there are also a substantial work in this topic, see $[1,2,6]$.

In literature, there are many attempts to generalize the concept of a group. For old studies and survey on algebraic theory of semi-groups, the reader can see the surveys [3]. One direction is the notion of LA-groups. The same thing for the concept of a ring. As far as we know, the concept of LA-rings is well known, see $[19,11]$.

People from Quaid-i-Azam university in Pakistan studied this notion. They gave a substantial contributions for the concepts of LA-semigroups and LAgroups. See the references $[8,9,10,12,13,15,16,18]$ for more details. In fact, we have seen a good efforts and well organized materials in Muhammed Sarwar's thesis [14]. In [17], we have seen some investigations of special class of semi-groups. Topological structures on LA-semi-groups appeared in [15].

However, the construction of tensor product of such algebraic structures is not exist in the literature. Another motivation for our work is that we believe that the non-commutative case occurs often in the applications of algebras in sciences and the topic of LA-modules is close to the commutative case.

Now we shall use the the paper [19] which deals with the notion of LAmodules. An LA-module over an LA-ring behaves like module and ring respectively. These concepts are very close to the commutative case. Consequently, we shall adapted the definition of the tensor product through the commutative case.

However, we need to consider some parallel results such as basis, direct sum decomposition and free modules. We have adapted standard approaches such as in [7, Theorem 4.1, Chapter 3] as well as in [5, Theorem 2.1, Chapter 4]. For the parallel results for the tensor product, we follow, in a verbatim way, the new book [4, Section 27, page 351].

We organize the paper as follows: In Section one, we recast the concept of LA-module, LA-ring and the related definitions such as LA-bilinear maps. Section 2 has been devoted to the new concept which we call it tensor product or LA-Tensor product as well as some parallel results to the ordinary case. The main result in this section is the existence of the tensor product of two LA-modules over an LA-ring. 


\section{LA-groups, LA-rings and LA-modules}

Let us start by recalling some definitions.

Definition 1.1 A groupoid $(M, \cdot)$ is called a left almost group (LA-group for short) if the following hold:

1. there exists an element $e \in M$ such that $e \cdot m=m$ for all $m \in M$.

2. for each $m \in M$, there exists $m^{\prime} \in M$ such that $m^{\prime} \cdot m=e$.

3. for each $m, n, k \in M$, we have $(m \cdot n) \cdot k=(k \cdot n) \cdot m$.

LA-groups exist and in general if $(M, \cdot)$ is an abelian group then $(M, *)$ is an LA-group, where the operation $*$ is defined in term of "." as follows: for all $m, n \in M, m * n:=n \cdot m^{-1}$. So, the integers with usual subtraction is an example of an LA-group.

The cancellation law holds in LA-groups and if $N$ is a non empty subset of an LA-group $M$ then $(N, \cdot)$ is an LA-subgroup of $M$ if $(N, \cdot)$ is itself an LA-group. In particular, A non-empty subset $N$ of an LA-group $M$ is an LA-subgroup of $M$ if and only if for all $n, n^{\prime} \in N$ we have $n \cdot n^{\prime} \in N$.

One can consider the left coset of an LA-subgroup $N$ of an LA-group $M$. There is an interesting property of LA-groups in such a way that if $N$ is an LA-subgroup of an LA-group $M$ then for all $x, y \in M$, we have $(x \cdot y) \cdot N=$ $N \cdot(y \cdot x)$. It follows that the set of all left coset of $N$ in $M$ namely $M / N$ is an LA-group. This holds without speaking of the concept of normal subgroup.

In general, an LA-group need not to be associative. There is a relationship between being abelain or associative. In fact, an LA-group is associative if and only if it is abelian. For more properties, see Chapter 5 in [14].

As in [19], we consider an LA-ring $R$ and an LA-group $M$. Then $M$ is an LA-module if there is an action from $R$ on $M$ in such a way the following hold:

- For all $m \in M$ and $r_{1}, r_{2} \in R$, we have $\left(r_{1}+r_{2}\right) m=r_{1} m+r_{2} m$.

- For all $m_{1}, m_{2} \in M$ and $r \in R$, we have $r\left(m_{1}+m_{2}\right)=r m_{1}+r m_{2}$.

- For all $m \in M$ and $r_{1}, r_{2} \in R$, we have $r_{1}\left(r_{2} m\right)=r_{2}\left(r_{1} m\right)$.

- For all $m \in M$ we have $1_{R} m=m$.

There are many examples which can be seen in the references mentioned above. 


\section{The construction of LA-Tensor product}

The aim of this work is to build the tensor product in the framework of LAmodules and LA-rings. Before starting such construction, we need some tools. One can see for non-abelian tensor product the crossed pairing maps as a tool. Also, for the ordinary tensor product, we often use bi-linear maps. So, let us define LA-bilinear map as follows.

The following definition is the cornerstone of our construction.

Definition 2.1 We consider an $L A$-ring $R$ and $M, N$ and $X$ be LA-modules over R. A map

$$
B: M \times N \longrightarrow X
$$

is said to be R-bilinear if and only if for every $m, m^{\prime} \in M, n, n^{\prime} \in N$ and $r \in R, B$ satisfies the following conditions:

- $B\left(m+m^{\prime}, n\right)=B(m, n)+B\left(m^{\prime}, n\right)$,

- $B(r m, n)=r \cdot B(m, n)$,

- $B\left(m, n+n^{\prime}\right)=B(m, n)+B\left(m, n^{\prime}\right)$,

- $B(m, r n)=r \cdot B(m, n)$.

Definition 2.2 Let $R$ be an LA-ring. Let $M$ and $N$ be two LA-modules over $R$. A tensor product of $M$ and $N$ over $R$ is an LA-module $M \otimes_{R} N$ which is equipped with an LA-bilinear map:

$$
\tau: M \times N \longrightarrow M \otimes_{R} N
$$

such that for each LA-module $X$ over $R$ and for each $R$-bilinear map

$$
\varphi: M \times N \longrightarrow X
$$

there is a unique LA-homomorphism

$$
\Phi: M \otimes_{R} N \longrightarrow X
$$

such that the following diagram

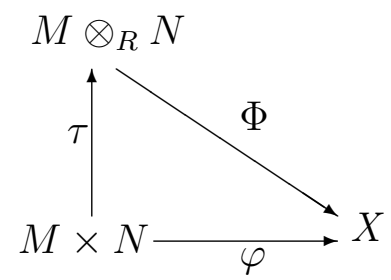

commutes, that is $\varphi=\Phi \circ \tau$. 
Now we would like to show that each or some properties of the usual tensor product hold in the new setting.

Proposition 2.3 Let $M$ and $N$ be two LA-modules over an LA-ring $R$. Tensor products of $M$ and $N$ over $R$ are unique up to unique isomorphism.

Proof: Let $T_{1}, T_{2}$ two tensor products of $M, N$ over $R$ which are equipped, respectively, with the $R$-bilinear maps

$$
\tau_{1}: M \times N \longrightarrow T_{1}, \tau_{2}: M \times N \longrightarrow T_{2}
$$

that satisfy Definition(2.3).

Since $T_{2}$ is an LA-module over $R$ and $\tau_{2}: M \times N \longrightarrow T_{2}$ is an $R$-bilinear map, then taking $X=T_{2}$ and $\tau_{2}: M \times N \longrightarrow T_{2}$ in place of $\varphi: M \times N \longrightarrow X$ in Definition (2.3), there exists a unique LA-homomorphism $\Phi_{1}: T_{1} \longrightarrow T_{2}$ such that the following diagram

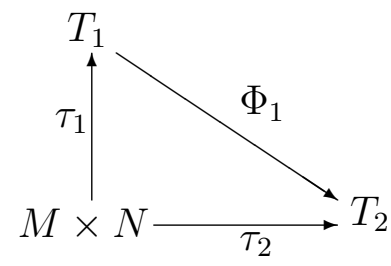

commutes, that is $\tau_{2}=\Phi_{1} \circ \tau_{1} \cdots(\star)$.

Similarly, reversing the roles, we can find also a unique LA-homomorphism $\Phi_{2}: T_{2} \longrightarrow T_{1}$ such that the following diagram

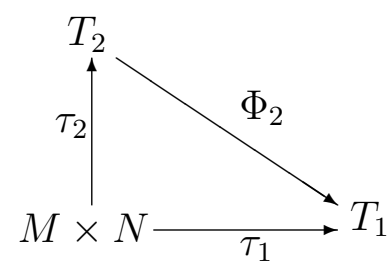

commutes, that is $\tau_{1}=\Phi_{2} \circ \tau_{2} \cdots(\star \star)$.

From $(\star)$ and $(\star \star)$, we get the following diagram

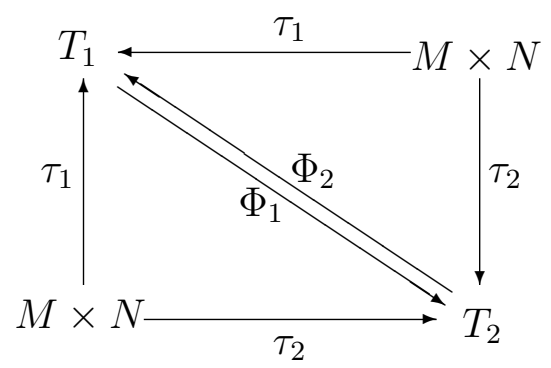


Since the maps $\Phi_{1}, \Phi_{2}$ are the unique maps that satisfy respectively $(\star)$ and $(\star \star)$, then the maps $\Phi_{1} \circ \Phi_{2}, \Phi_{2} \circ \Phi_{1}$ are the unique maps that satisfy respectively the following equalities:

$$
\tau_{1}=\Phi_{2} \circ \Phi_{1} \circ \tau_{1} \quad, \quad \tau_{2}=\Phi_{1} \circ \Phi_{2} \circ \tau_{2} .
$$

But the identity map satisfies also the above equalities. Hence, the maps

$$
\Phi_{2} \circ \Phi_{1}: T_{1} \longrightarrow T_{1}, \Phi_{1} \circ \Phi_{2}: T_{2} \longrightarrow T_{2}
$$

are necessarily equal to the identity map. Therefore, the maps $\Phi_{1}$ and $\Phi_{2}$ are mutual inverses.

\section{LA-Free modules and some parallel results}

Let $R$ be an LA-ring with left identity $1_{R}$. Let $A$ be an LA-module over the LA-ring $R$. Let $X$ be a subset of $A$. Then we say that $X$ is linearly independent set over $R$ or (LA- linearly independent over $R$ ) if for any choice of distinct elements $x_{1}, x_{2}, \cdots, x_{n}$ from $X$ and $r_{1}, r_{2}, \cdots, r_{n}$ from $R$ it holds that $\sum_{i=1}^{i=n} r_{i} x_{i}=0$ implies $r_{i}=0$ for all $i$. Now if $A$ is generated by the set $X$ then we say that $X$ spans $A$.

The following definition of LA-basis is the starting point to consider the notion of LA-free module.

Definition 3.1 A linearly independent subset of the LA-module $A$ that spans $A$ is called a basis of $A$ or (LA-basis).

The proof of the following proposition is similar to that in the ordinary case in [5, Theorem 2.1, Chapter 4].

Proposition 3.2 Let $R$ be an LA-ring with left identity and $A$ be an LAmodule which is unitary ( that is $1_{R} a=a$ for all $a \in A$ ). Then the following are equivalent:

1. A has a non-empty LA-basis.

2. There exists a non-empty set $X$ and a function $e: X \rightarrow A$ with the following universal property: Given any unitary $L A$-module $B$ and a function $f: X \rightarrow B$ there is a unique LA-homomorphism $\bar{f}: A \rightarrow B$ such that $\bar{f} \circ e=f$.

Definition 3.3 A unitary LA-module $F$ over an LA-ring $R$ which satisfies any condition of the above proposition is called an LA-free module on the set $X$. 
Proposition 3.4 Let $M$ and $N$ be two LA-modules over an LA-ring $R$. The tensor product of $M$ and $N$ over $R$ exists.

Proof: Let $M$ and $N$ be two LA-module over $R$ and let $F$ be an LA-free module of $M \times N$ with a canonical basis $\{e(m, n)\}_{(m, n) \in M \times N}$. We consider the LA-submodule $Y$ of $F$ generated by all elements of $F$ with form:

- $V_{1}\left(m+m^{\prime}, n\right)=e\left(m+m^{\prime}, n\right)-e(m, n)-e\left(m^{\prime}, n\right)$,

- $V_{2}(r m, n)=e(r m, n)-r \cdot e(m, n)$,

- $V_{3}\left(m, n+n^{\prime}\right)=e\left(m, n+n^{\prime}\right)-e(m, n)-e\left(m, n^{\prime}\right)$,

- $V_{4}(m, r n)=e(m, r n)-r \cdot e(m, n)$,

for all $r \in R, m, m^{\prime} \in M$ and $n, n^{\prime} \in N$.

We try to show that the tensor product $M \otimes N$ of $M$ and $N$ is exactly the quotient module $F / Y$. According to our construction, $F / Y$ is an LA-module over $R$. Define the map $\pi$ as follow:

$$
\begin{aligned}
\pi: & F \longrightarrow F / Y \\
& m \longmapsto \pi(m)=Y+m
\end{aligned}
$$

Then we deduce that $\pi$ is a surjective LA-group homomorphism. So, the map

$$
\tau=\pi \circ e: M \times N \longrightarrow F / Y
$$

define a tensor product of $M$ and $N$ over $R$. In fact, depending to the structure of the submodule $Y$ defined above one can easily show that $\tau$ is an LA-bilinear map.

Given now an LA-module $X$ over $R$ and an LA-bilinear map $\varphi: M \times N \longrightarrow X$. According to the universal property of LA-free modules, there exists a unique LA-module homomorphism $\psi: F \longrightarrow X$ such that $\varphi=\psi \circ e$, that means the following diagram commutes

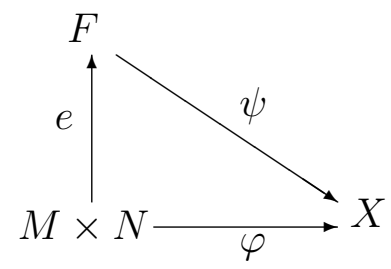

In the other hand, for all $m, m^{\prime} \in M$ and $n, n^{\prime} \in N$ we have

$$
\psi\left(V_{1}\left(m+m^{\prime}, n\right)\right)=\psi\left(e\left(m+m^{\prime}, n\right)-e(m, n)-e\left(m^{\prime}, n\right)\right) .
$$


Since, $\psi$ is a linear map and $\varphi$ is bilinear, then

$$
\begin{aligned}
\psi\left(V_{1}\left(m+m^{\prime}, n\right)\right) & =\psi\left(e\left(m+m^{\prime}, n\right)\right)-\psi(e(m, n))-\psi\left(e\left(m^{\prime}, n\right)\right) \\
& \left.=\psi \circ e\left(m+m^{\prime}, n\right)-\psi \circ e(m, n)-\psi \circ e\left(m^{\prime}, n\right)\right) \\
& \left.=\varphi\left(m+m^{\prime}, n\right)-\varphi(m, n)-\varphi\left(m^{\prime}, n\right)\right) \\
& =0 .
\end{aligned}
$$

Following the same argument, we prove that

$$
\psi\left(V_{2}(r m, n)\right)=\psi\left(V_{3}\left(m, n+n^{\prime}\right)\right)=\psi\left(V_{4}(m, r n)\right)=0,
$$

that is

$$
V_{1}\left(m+m^{\prime}, n\right), V_{2}(r m, n), V_{3}\left(m, n+n^{\prime}\right), V_{4}(m, r n) \in \operatorname{ker} \psi
$$

for all $r \in R, m, m^{\prime} \in M$ and $n, n^{\prime} \in N$. But $Y$ is an LA-submodule of $F$ generated by the elements of the set

$\left\{V_{1}\left(m+m^{\prime}, n\right), V_{2}(r m, n), V_{3}\left(m, n+n^{\prime}\right), V_{4}(m, r n) ; \quad r \in R, m, m^{\prime} \in M, n, n^{\prime} \in N\right\}$,

thus $Y \subset \operatorname{ker} \psi$. According to the theorem of factorization of homomorphism, $\psi$ factors through $F / Y$, that is, there is an LA-homomorphism $\Phi: F / Y \longrightarrow$ $X$, such that $\psi=\Phi \circ \pi: F \longrightarrow X$. So, the uniqueness of $\psi$ and the surjection of $\pi$ yield to the uniqueness of $\Phi$. Indeed, for two maps $\Phi_{1}$ and $\Phi_{2}$ with $\Phi_{1} \circ \pi=\psi=\Phi_{2} \circ \pi$, given $x \in F / Y$, since $\pi$ is surjective, there exists $y \in F$, such that $\pi(y)=x$. Then,

$$
\Phi_{1}(x)=\Phi_{1} \circ \pi(y)=\psi(y)=\Phi_{2} \circ \pi(y)=\Phi_{2}(x) .
$$

Thus, $\Phi_{1}=\Phi_{2}$.

We remark that Proposition 2.3 guarantees the existence and the uniqueness of the LA-tensor product.

Let $M \otimes_{R} N$ be the tensor product of two LA-modules $M$ and $N$ over an LA-ring $R$ defined by the map $\tau: M \times N \longrightarrow M \otimes N$. We denote by $m \otimes n$ the image of $(m, n) \in M \times N$ under the map $\tau$. Then, $m \otimes n$ is called a monomial tensor. Through the behavior of the monomial tensors, we try to characterize the LA-module $M \otimes_{R} N$ and we get the following result:

Proposition 3.5 Let $M$ and $N$ be two LA-modules over an LA-ring $R$. The tensor product $M \otimes_{R} N$ of $M$ and $N$ over $R$ is generated by the set of all the monomial tensors $m \otimes n$ with $m \in M$ and $n \in N$.

Proof: Let $X$ be the LA-submodule of $M \otimes_{R} N$ generated by the set of all the monomial tensors $m \otimes n$ with $m \in M$ and $n \in N$. We prove that $X=M \otimes_{R} N$. 
Put $Y=M \otimes_{R} N / X$ and let $\pi: M \otimes_{R} N \longrightarrow Y$ be the canonical surjection. We consider the map $\varphi$ defined as follow:

$$
\begin{aligned}
& \varphi: M \times N \quad \longrightarrow \quad Y \\
& (m, n) \longmapsto \varphi(m, n)=\overline{0} \text {. }
\end{aligned}
$$

Since $\varphi$ is $R$-bilinear, then according to Definition(2.3), there exists a unique LA-homomorphism $\Phi: M \otimes N \longrightarrow Y$ such that

$$
\varphi=\Phi \circ \tau
$$

where $\tau: M \times N \longrightarrow M \otimes N$ is the map that defined the tensor product of $M$ and $N$. It is clair that the 0-map $\pi: M \otimes_{R} N \longrightarrow Y$ satisfies the equation(1). On the other hand, for any $(m, n) \in M \times N$, we have

$$
\tau(m \times n)=m \otimes n \in X .
$$

Therefore

$$
\pi \circ \tau(m, n)=\pi(m \otimes n)=0=\varphi(m, n) .
$$

That means, the map $\pi$ satisfies also the equation(1). Then, by the uniqueness of the map $\Phi$, we get $\Phi=\pi=0$. But $\pi$ is surjective, then we have $Y=$ $\pi(M \otimes N)=\{0\}$. That is $M \otimes N=X$.

Proposition 3.6 Let $M$ and $N$ be two LA-modules over an LA-ring $R$ generated respectively by the sets $\left\{m_{\alpha} \in M ; \alpha \in A\right\},\left\{n_{\beta} \in N ; \beta \in B\right\}$. Then, the tensor product $M \otimes_{R} N$ of $M$ and $N$ over $R$ is generated by the set

$$
\left\{m_{\alpha} \otimes n_{\beta} \in M \otimes N ;(\alpha, \beta) \in A \times B\right\} .
$$

Proof: Since monomial tensors generate the tensor product, it suffices to show that every monomial tensor is expressible in terms of $m_{\alpha} \otimes n_{\beta}$. Let $m \in M, n \in N$, then

$$
m=\sum_{\alpha \in A} r_{\alpha} m_{\alpha} \quad \text { and } \quad n=\sum_{\beta \in B} s_{\beta} n_{\beta}
$$

where $r_{\alpha}, s_{\beta} \in R$ for all $(\alpha, \beta) \in A \times B$. Since the tensor product is defined by a bilinear map, then we have:

$$
m \otimes n=\left(\sum_{\alpha \in A} r_{\alpha} m_{\alpha}\right) \otimes\left(\sum_{\beta \in B} s_{\beta} n_{\beta}\right)=\sum_{(\alpha, \beta) \in A \times B} r_{\alpha} s_{\beta} m_{\alpha} \otimes n_{\beta} .
$$




\section{Functorial properties of LA-modules}

Now we discuss the tensor product of LA-module homomorphisms:

Let $f: M \rightarrow M^{\prime}$ and $g: N \rightarrow N^{\prime}$ be two LA-module homomorphisms over an LA-ring $R$. We would like to define and study the tensor product map

$$
f \otimes g: M \otimes N \rightarrow M^{\prime} \otimes N^{\prime}
$$

The natural definition for the above map is by the rule:

$$
f \otimes g(m \otimes n)=f(m) \otimes g(n),
$$

for all $m \in M$ and all $n \in N$.

Now it is not difficult to show that $f \otimes g$ is well-defined. In fact, this map is the unique LA-module homomorphism which is compatible with the definition of the LA-tensor product $M \otimes N$.

Let us parallel some results in the ordinary tensor product in the case of LA-module homomorphisms.

Proposition 4.1 Let $f: M \rightarrow M^{\prime}$ and $g: N \rightarrow N^{\prime}$ be two LA-module homomorphisms over an LA-ring $R$. Then we have:

- If $f$ and $g$ are onto then $f \otimes g$ is onto.

- If $f$ and $g$ are onto then we have

$$
\operatorname{kernel}(f \otimes g)=\operatorname{kerenl}(f) \otimes N+M \otimes \operatorname{kernel}(g) .
$$

- If $R$ is commutative $L A$-ring and $I$ and $J$ are ideals of $R$. Then there is an LA-module isomorphism $R / I \otimes R / J \cong R /(I+J)$.

The proof of the following theorem is straightforward from the definition.

Theorem 4.2 If $R$ is an LA-ring with identity and $M$ is a unitary LAmodules over $R$. Then there is an LA-isomorphism $M \otimes R \cong M$.

\section{References}

[1] A. M. Alghamdi and F. G. Russo, Remarks on the relative tensor degree of finite groups, accepted in Filomat (2014).

[2] R. Brown, D. L. Johnson, E. F. Robertson, Some computations of nonabelian tensor products of groups, J. Algebra 111 (1987), 177-202.

[3] A. H. Clifford, G. B. Preston, Algebraic theory of Semigroups, Amer. Math. Soc. Math. Surveys, 7, V. I, II, 1961, 1967. 
[4] P. Garrett, Abstract Algebra, June 2007, Minneapolis: www.math.umn.edu/garrett/m/algebra/notes/Whole.pdf.

[5] T. W. Hungerford, Algebra, Springer-Verlag 1974.

[6] N. Inassaridze, Nonabelian tensor products and Nonabelian homology of groups, Journal of Pure and Applied Algebra 112 (1996), 191-205.

[7] S. Lang, Algebra, Graduate texts in Mathematics, GTM 211 Springer 2002 .

[8] K. Madad, A. Venus, Faisal, Ideals in Intra-Regular Left Almost Semigroups, arXiv:1012.5598.

[9] K. Madad, A. Tauseef, Characterizations of Intra-Regular Left Almost Semigroups by Their Fuzzy Ideals, Journal of Mathematics Research Vol. 2, No. 3; August 2010: www.ccsenet.org/jmr

[10] A. Muhammad, S. Muhammad, Y. Naveed, Roughness in left almost semigroups, Journal of Advanced Research in Pure Mathematics, Vol. 3, Issue. 3, 2011, pp. 70-88.

[11] S. Muhammed, On Left Almost Ring, Proc. of Yth International Pure Math. Conference (2007).

[12] S. Muhammad, Structutal Properties of LA-Semi-groups, M. Phil. Dissertaton, Quaid-i-Azam University, Islamabad, 1987.

[13] S. Muhammad, S. Tariq, Some Basic Properties of LA-Ring, International Mathematical Forum, Vol. 6, 2011, no. 44, 2195-2199.

[14] S. Muhammad, Conditions for LA-Semi-groups to resemble associative structure, Ph. D. Thesis, Quaid-i-Azam University, Islamabad, 1993.

[15] Q. Mushtaq, K. Madad, P. S. Kar, Topological Structures on LASemigroups, Bull. Malays. Math. Sci. Soc. (2) 36(4) (2013), 901906.

[16] Q. Mushtaq, K. Madad, Ideals in left almost semigroups, arXiv:0904.1635.

[17] Q. Mushtaq, Zeroids and idempoids in AG-groupoids, Quasigroups and Related Systems 11 (2004), 79-84.

[18] S. Tariq, R. Fazal, R. Muhammad, On Near Left Almost Rings, International Mathematical Forum, Vol. 6, 2011, no. 23, 1103 - 1111

[19] S. Tariq, R. Muhammad, A. Gauhar, On LA-Modules, Int. J. Contemp. Math. Sciences, Vol. 6, 2011, no. 21, 999 - 1006.

Received: May 3, 2014 